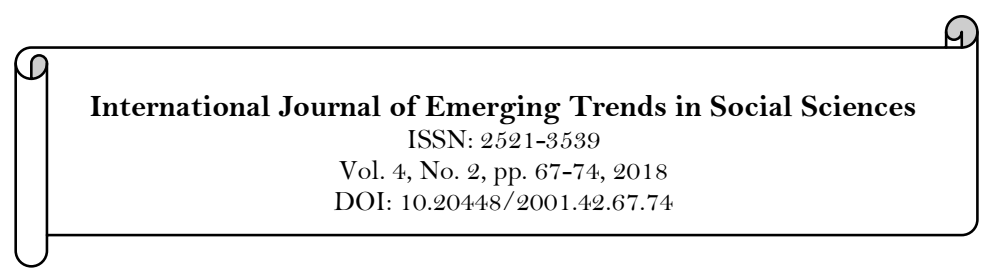

\title{
Optimal Bid Strategies in Crowdsourcing Contest Based on Multi-Attribute Auctions
}

\author{
Qi Xus \\ Huixian Zhang ${ }^{2}$ \\ ${ }^{1,2}$ Glorious Sun School of Business and Management, Donghua University, Shanghai 20005 1, China.
}

\begin{tabular}{l|l} 
Abstract & \\
$\begin{array}{l}\text { As a business model under the emerging social interaction behavior, } \\
\text { crowdsourcing gather public knowledge, skills, and information to }\end{array}$ \\
help companies directly face consumers, fully explore innovative \\
product design and sales markets, and make full use of Internet \\
Multiple attributes \\
advantages to respond market demand. This paper explores \\
crowdsourcing contest which is under a fixed bonus mechanism. The \\
purpose of crowdicipants \\
All pay auction.
\end{tabular}

\section{Introduction}

With the rapid development of the Internet, an emerging business model emerged, such as crowdsourcing. Crowdsourcing is not only rapidly penetrating in research and development, market development and other fields, but also widely used in creative product design, advertising design, marketing strategies and so on. Companies are innovating through crowdsourcing models. Through this model, the task which should be completed by the company's employees will be handed over to the public through the Internet. Innovating through crowdsourcing contest can not only reduce the cost of innovation but also promote the company. For instance, in order to develop a better collaborative filtering algorithm, Netflix hold a crowdsourcing competition offering a million dollars to the contestant who can develop a better collaborative filtering algorithm.

Crowdsourcing contest can be modeled as multi-attribute reverse auction, because the crowdsourcing wants to evaluate the bid submitted by the contestant in many aspects. David, Azoulay-Schwartz, and Kraus (2002) builds a procurement multi-attribute auction model and provide the best strategy for buyers and sellers. However, some of the parameters in the model are not adapted to the actual economic activities. Some scholars have optimized the model

In order to maximize the utility of both the contestant and the crowdsourcing, various scholars have studied the participation strategies of both parties. For the crowdsourcing, many scholars have studied their reward strategies, entry fee strategies, information feedback strategies, and the number of participants; for the contestant, many scholars study the bidding strategy of the contestant. Archak and Sundararajan (2009) studied the bidding strategy of different risk preference contestant and the bonus distribution strategies of the crowdsourcing for different risk preferences. Moldovanu and Sela (2001) studied the bidding strategy of the contestant and the bonus distribution strategy of the crowdsourcing under the condition that the cost function of the acceptor is linear and nonlinear.

Yang, Chen, and Pavlou (2009) through research on second-hand data, it is found that the higher the bonus amount set by the crowdsourcing, the more contestants to participate in the crowdsourcing contest. The greater the number of crowdsourcing contest, the more intense the competition, so that the quality of the 
bid submitted by the contestants can be significantly improved. And Taylor (1995) found that an increase in the number of contestants would increase the intensity of the crowdsourcing contest, but this would reduce the probability of winning and increase the time, experience and communication costs to review the bid. So , Che and Gale (2003) recommended to reduce the number of participants to 2. And Morgan and Wang (2010) think setting entry fee can limit the number of people participating in the crowdsourcing contest. But Terwiesch and $\mathrm{Xu}(2008)$ found that for creative competitions and experimental competitions, more answerers can increase the diversity of solutions, thereby increasing the publisher's revenue. It is the best choice without charging admission fees. Schöttner (2008) doesn't think that admission fees should be charged in order to avoid the platform or the crowdsourcing seeking personal gain.

In summary, more research has focused on the issues related to crowdsourcing contest under a single attribute of a single crowdsourcing task. However, in order to get high-quality solutions in crowdsourcing contest, how to design effective incentives to attract potential contestants, maximize the interests of crowdsourcing parties and participants, and enhance the innovation ability of enterprises. Important issues that need to be addressed in creative crowdsourcing. Chawla, Hartline, and Sivan (2015) considers that the utility of the crowdsourcing is the best effort of all contestants and the sum of the efforts of all contestants. The former is more used in research and development competitions, design competitions, etc.; the latter is mainly for brainstorming and broadening ideas, the utility of all the crowdsourcing in this paper adopts the first type. In addition, unlike previous scholars' research on crowdsourcing contest, this paper uses the relevant theory of multi-attribute reverse auction, analyzes the quality attributes of bid, establishes the utility model of both the contestants and the crowdsourcing, and studies the bidding strategy of the contestant. The bonus strategy of the crowdsourcing and the expected utility of both the contestant and the crowdsourcing.

\section{Model}

In the crowdsourcing contest, firstly the crowdsourcing announces the requirements for bid, the scoring rules, the minimum score $S$ min for the program and the size of the bonus through the crowdsourcing website. And then the contestant designs the bid according to the scoring rules and their own utility and submit. Finally, the crowdsourcing calculates the score of each creative plan according to the scoring rule, and the highest scored contestant receives the bonus. The crowdsourcing website issues bonuses to the winners.

In order to facilitate the study, the following assumptions are given:

Assumption 1. The crowdsourcing and contestants involved in crowdsourcing are rational and the preferences for the various attributes of the bid are relatively independent.

Assumption 2. The each attributes of the bid are independent, that is, the value of one attribute does not depend on the value of other attributes

Assumption 3. The utility function of the crowdsourcing meets the conditions: $\frac{\partial U_{c}}{\partial q_{j}}>0$ 、 $\frac{\partial^{2} U_{c}}{\partial q_{j}{ }^{2}}<0$ 、 $\lim _{q_{j} \rightarrow \infty} \frac{\partial U_{c}}{\partial q_{j}}=0$ and $\lim _{q_{j} \rightarrow 0} \frac{\partial U_{c}}{\partial q_{j}}=\infty$, That is, the utility of each attribute of the bid to the crowdsourcing is incremental, but the marginal utility is diminishing. The cost function of the contestant meets the conditions: $\frac{\partial C\left(\theta, q_{j}\right)}{\partial q_{j}}>0, \frac{\partial^{2} C\left(\theta, q_{j}\right)}{\partial q_{j}{ }^{2}} \geq 0$ and $\frac{\partial^{2} C\left(\theta, q_{j}\right)}{\partial q_{j} \partial \theta_{i}} \geq 0$. That is, the increase in the effort of the contestant on the attributes of the bid, and the cost will increase.

Assumption 4. Each contestant's cost parameter $\theta$ is a private information. The crowdsourcing knowns only the distribution function of the cost parameter. It is assumed that $\theta$ is independently and identically distributed uniform distribution over $[\underline{\theta}, \bar{\theta}]$, according to a distribution function $\Phi(\theta)$, continuously differentiable density $\varphi(\theta)$.

\subsection{Cost Function of the Contestant}

The contestant will pay for his own ideas and abilities in order to obtain the crowdsourcing bonus, and the contestant will generate the cost. The higher the contestant's cost parameter, the more effort is needed to realize a particular quality level. When the cost parameter of the contestant is its upper bound $\bar{\theta}$, the bidding strategy and the utility of the contestant is zero. 
When contestant with cost parameter $\theta$ provides bid with quality attributes $q_{1}, \ldots, q_{k}, \ldots, q_{m}$, the cost of the contestant is

$$
C\left(\theta, q_{k}\right)=\theta \sum_{k=1}^{m} a_{k} q_{k}^{t_{k}}
$$

The cost parameter $\theta$ is independently and identically distributed uniform distribution over $[\underline{\theta}, \bar{\theta}]$,according to a distribution function $\Phi(\theta)$, continuously differentiable density $\varphi(\theta) ; a_{k}$ is the cost coefficient assigned to k-th attribute by the contestant, and $a_{k}>0 ; q_{k}$ is the value of the k-th quality attribute of the bid; in order to ensure the marginal cost of the contestant is increasing, so set $t_{k}>1$.

\subsection{Utility Function of the Contestant}

The contestant with the highest effort wins the prize $M$. Crowdsourcing contest is similar to all pay auction, if the contestant wins, it can get positive income. If the contestant can't win, then the effort can't get any return. The contestant who wins the first place in the crowdsourcing contest will receive all the bonus, so if contestant wins, its utility function is

$$
U_{a}(\theta, b)=M-C\left(\theta, q_{k}\right)=M-\theta \sum_{k=1}^{m} a_{k} q_{k}^{t_{k}}
$$

If the contestant does not win the first place, its utility function is

$$
U_{a}(\theta, b)=-\theta \sum_{k=1}^{m} a_{k} q_{k}^{t_{k}}
$$

2.3. Utility Function of the Crowdsourcing

The utility function of the crowdsourcing is

$$
U_{c}=\sum_{k=1}^{m} w_{k} q_{k}^{s_{k}}-M
$$

$w_{k}$ is the weight given to the k-th attribute assigned by the crowdsourcing, it indicates the degree of preference for different attributes published by the crowdsourcing. The utility of the crowdsourcing is increasing, and the marginal utility is diminishing, so $0<s_{k}<1$.

\subsection{Scoring Function of Crowdsourcing}

Compared with the single attribute evaluation, the multi-attribute evaluation of the bid is mainly reflected in the judgment of multiple aspects of the submitted bid when determining the winner. Because the bidder's bid needs to be evaluated from multiple attributes, it is necessary to develop a scoring function according to the bidder's preference for bidding, so that the bid submitted by the contestant can be comprehensively evaluated to maximize its utility. Therefore, the scoring function can be determined by its utility function. In order to maximize its own utility, the scoring rules published by the crowdsourcing may be different from the real utility function. This difference can be achieved by the difference between the attribute weights $W_{k}$ published by the crowdsourcing in the scoring function and the attribute weights $w_{k}$ reflecting the true preference in the utility function.

$$
S_{b}=\sum_{k=1}^{m} W_{k} q_{k}^{r_{k}}
$$

\section{Optimal Strategy for Both Parties \\ 3.1. Optimal Bidding Strategy for the Contestant}

In the crowdsourcing contest, the bidding strategy of the contestant is to determine the optimal attribute values to maximize his utility according to the scoring function published by the crowdsourcing and its own income function. 
Adding the quality attribute value of the bid to the crowdsourcing contest will increase its cost, but if the quality attribute of the bid is reduced, the probability of winning will be reduced, so it is necessary to optimize the attribute value of the bid.

The probability that the contestant gets the first place is $p_{\text {win }}(\theta)=(1-\Phi(\theta))^{N-1}$, so contestant's Expected utility is

$$
\begin{aligned}
& E U\left(\theta, q_{1}, q_{2}, \ldots, q_{k}\right)=M p_{\text {win }}(\theta)-c\left(\theta, q_{1}, q_{2}, \ldots, q_{k}\right) \\
& =M(1-\Phi(\theta))^{N-1}-\theta \sum_{k=1}^{m} a_{k} q_{k}^{t_{k}}
\end{aligned}
$$

the first-order conditions (FOC) is as follow:

$$
\begin{aligned}
& \frac{\partial E U\left(\theta, q_{1}, q_{2}, \ldots, q_{m}\right)}{\partial q_{k}}=\frac{\partial}{\partial q_{k}}\left[M\left(1-\Phi\left(q_{k}^{-1}(\theta)\right)\right)^{N-1}-\theta \sum_{k=1}^{m} a_{k} q_{k}^{t_{k}}\right] \\
& =-M(N-1)\left(1-\Phi\left(q_{k}^{-1}(\theta)\right)\right)^{N-2} \frac{\varphi(\theta)}{q_{k}{ }^{\prime}(\theta)}-\theta a_{k} t_{k} q_{k}{ }^{\left(t_{k}-1\right)}=0
\end{aligned}
$$

By solving the above equation, the following solution is gained:

$$
q_{k}^{*}=\left(\frac{M(N-1)}{a_{k}} \int_{\theta}^{\bar{\theta}} \frac{1}{t} \varphi(t)(1-\Phi(t))^{N-2} d t\right)^{\frac{1}{t_{k}}}
$$

According to the hypothesis, the cost parameters of the contestants satisfies uniform distribution, the k-th attribute value of the contestant is affected by the contestant's own cost parameter $\theta$ and the quality attribute cost coefficient of the bid $a_{k}$, so the optimal bidding strategy for the k-th attribute is:

$$
q_{k}^{*}(\theta)=\left(\frac{M(N-1)}{a_{k}} \int_{\theta}^{\bar{\theta}} \frac{(\bar{\theta}-t)^{N-2}}{t(\bar{\theta}-\underline{\theta})^{N-3}} d t\right)^{\frac{1}{t_{k}}}
$$

It can be seen from Equation (6), for the bid with multiple attributes, the value of each attribute is related to the amount of the bonus announced by the crowdsourcing, the cost parameter of the contestant and the number of participants in the competition.

\subsubsection{Expected Utility of the Contestant}

The contestant bids with the optimal bid combination $\left(q_{1}^{*}, q_{2}^{*}, \ldots, q_{k}^{*}\right)$. At this point, the expected utility of the contestant is

$$
E U_{a}\left(q_{1}^{*}(\theta), q_{2}^{*}(\theta), \ldots, q_{k}^{*}(\theta)\right)=M(1-\Phi(\theta))^{n-1}-\theta \sum_{k=1}^{m} a_{k} q_{k}^{*}(\theta)^{t_{k}}
$$

According to the hypothesis, the cost parameters of the contestants satisfies uniform distribution. Therefore, the expected utility of the contestant is

$$
E U(\theta)_{a}=M\left(\frac{\bar{\theta}-\theta}{\bar{\theta}-\underline{\theta}}\right)^{N-1}-M \theta(N-1) m \int_{\theta}^{\bar{\theta}} \frac{1}{t(\bar{\theta}-\underline{\theta})}\left(1-\frac{t-\underline{\theta}}{\bar{\theta}-\underline{\theta}}\right)^{N-2} d t
$$

It is known from the expected utility expression of the contestant that the expected utility of the contestant is independent of the cost coefficient of each attribute, and is only related to the cost parameter of the contestant, the number of participants in the competition, and the bonus.

\subsubsection{Score of the contestant}

In the crowdsourcing contest, in order to prevent the contestant from joining to provide bids with the low quality, so the crowdsourcing sets the minimum score $S$ when the crowdsourcing contest task is announced. The contestant compares the score of his bid with the minimum score required by the crowdsourcing, if $S_{b}>S$, the contestant will participate in the crowdsourcing contest. Therefore, each contestant needs to calculate his own score before participating in the competition. 
Take formula (6) into formula (5), the score of the contestant is

$$
\operatorname{Maxscore}(\theta)=\sum_{k=1}^{m} W_{k} q_{k}^{r_{k}}=\sum_{k=1}^{m} W_{k}\left(\frac{M}{a_{k}}(N-1) \int_{\theta}^{\bar{\theta}} \frac{(\bar{\theta}-t)^{N-2}}{t(\bar{\theta}-\underline{\theta})^{N-3}} d t\right)^{\frac{r_{k}}{t_{k}}}
$$

In the crowdsourcing contest, the crowdsourcing sets the minimum score for bid. For the crowdsourcing, on the one hand, it can prevent the contestant from joining to provide bids with the low quality; on the other hand, it can help the crowdsourcing to filter out some of the bids with lower quality. For the contestant, it can help contestant make decisions about whether to participate in the crowdsourcing contest.

\subsubsection{Related Factor Analysis}

(1) The cost coefficient of each attribute affects the bid value of the attribute

In order to study the effect of cost coefficient on quality attribute values, take the first derivative of formula (6) with respect to $a_{k}$,

$$
\frac{\partial q_{k}{ }^{*}(\theta)}{\partial a_{k}}=-\frac{M}{a_{k}{ }^{2} t_{k}}(N-1) \int_{\theta}^{\bar{\theta}} \frac{(\underline{\theta}-t)^{N-2}}{t(\bar{\theta}-\underline{\theta})^{N-3}} d t\left(\frac{\left(1-c_{s}\right) M(N-1)}{a_{k}} \int_{\theta}^{\bar{\theta}} \frac{(\underline{\theta}-t)^{N-2}}{t(\bar{\theta}-\underline{\theta})^{N-3}} d t\right)^{\frac{1}{t_{k}}-1}
$$

Observing the above formula $\frac{\partial q_{k}{ }^{*}(\theta)}{\partial a_{k}}<0$. Therefore, the greater the cost coefficient of the attribute, the less effort the contestant will make on the attribute.

(2) The effect of the cost parameter on the bid value of the attribute

In order to study the effect of cost parameter on quality attribute values, take the first derivative of formula (6) with respect to $\theta$,

$$
\frac{\partial q_{k}(\theta)}{\partial \theta}=-\frac{M(N-1)}{a_{k} \theta t_{k}(\bar{\theta}-\underline{\theta})}\left(\frac{\bar{\theta}-\theta}{\bar{\theta}-\underline{\theta}}\right)^{N-2}\left(\frac{M(N-1)}{a_{k}} \int_{\theta}^{\bar{\theta}} \frac{1}{t(\bar{\theta}-\underline{\theta})}\left(\frac{\bar{\theta}-t}{\bar{\theta}-\underline{\theta}}\right)^{N-2} d t\right)^{\left(\frac{1}{t_{k}}-1\right)}
$$

Observing the above formula $\frac{\partial q_{k}(\theta)}{\partial \theta}<0$, it can be seen that the higher the cost parameter of the contestant, the less effort the contestant will make on the attribute. This seems to be inconsistent with common sense, but actually in this mode where the first contestant gets all the bonuses, a contestant with a lower cost parameter means that it is more capable and has higher expectations for the quality of the bid submitted by itself, so more effort will be put into bidding for each attribute.

\section{(4) The impact of the cost parameter of the contestant on its expected utility}

In order to study the effect of cost parameter on its expected utility, take the first derivative of formula (8) with respect to $\theta$,

$$
\begin{aligned}
& \frac{\partial E U(\theta)_{a}}{\partial \theta}=-M(N-1)\left(\frac{\bar{\theta}-\theta}{\bar{\theta}-\underline{\theta}}\right)^{N-2}-(N-1) M \int_{\theta}^{\bar{\theta}} \frac{1}{t(\bar{\theta}-\underline{\theta})}\left(1-\frac{t-\underline{\theta}}{\bar{\theta}-\underline{\theta}}\right) d t \\
& -(N-1) \theta M \frac{1}{\theta(\bar{\theta}-\underline{\theta})}\left(1-\frac{\theta-\underline{\theta}}{\bar{\theta}-\underline{\theta}}\right)^{N-2} \\
& =M(N-1)\left(\frac{\bar{\theta}-\theta}{\bar{\theta}-\underline{\theta}}\right)^{N-2}\left(-\frac{1}{\bar{\theta}-\underline{\theta}}-1\right)-(N-1) M \int_{\theta}^{\bar{\theta}} \frac{1}{t(\bar{\theta}-\underline{\theta})}\left(1-\frac{t-\underline{\theta}}{\bar{\theta}-\underline{\theta}}\right)^{N-2} d t
\end{aligned}
$$


Observing the above formula $\frac{\partial E U(\theta)_{a}}{\partial \theta}<0$, the higher the cost parameter of the contestant, the lower the expected utility. This can promote the contestant to continuously improve their abilities to make them more competitive int the contest.

\subsection{Optimal Strategy of the Crowdsourcing}

The highest quality contestant submitted in the crowdsourcing contest receives the bonus. In order to maximize the expected utility, the crowdsourcing must consider setting the bonus amount.

\subsubsection{Expected Utility of the Crowdsourcing}

The expected utility of the crowdsourcing is the sum of the quality of the bid submitted by the contestant. This paper use $S P$ to express the expected utility of the crowdsourcing. According to formula (4), the expected utility of the crowdsourcing can be expressed as

$$
S P=N \int_{\underline{\theta}}^{\bar{\theta}}\left(\sum_{k=1}^{m} w_{k}\left(q_{k}^{*}(t)\right)^{s_{k}}-M\right) \varphi(t) d t
$$

Take formula (6) into formula (9), the expected utility of the crowdsourcing can be expressed as

$$
S P=\frac{N}{\bar{\theta}-\underline{\theta}} \int_{\underline{\theta}}^{\bar{\theta}}\left(\sum_{k=1}^{m} w_{k}\left(\frac{M(N-1)}{a_{k}} \int_{\theta}^{\bar{\theta}} \frac{1}{t(\bar{\theta}-\underline{\theta})}\left(\frac{\bar{\theta}-t}{\bar{\theta}-\underline{\theta}}\right)^{N-2} d t\right)^{\frac{s_{k}}{t_{k}}}-M\right) d \theta
$$

It can be seen that the size of the bonus amount, the number of contest participants, the weight given to each attribute by the crowdsourcing, and the distribution of the ability of the contestant affect the utility of the crowdsourcing.

\subsubsection{Bonus Strategy}

Under the fixed reward mechanism, the crowdsourcing can set the appropriate bonus amount to highlight the advantages of the crowdsourcing contest. Because the increase in the amount of bonuses can encourage contestants to make more efforts, but it also increases their own costs, this paper will next study the impact of the size of the bonus on the utility of the crowdsourcing.

In order to maximize the utility of the crowdsourcing, take the first derivative of formula (10) with respect to $M$, make the derivative equals to zero, and solve the equation. Then we can get formula (11).

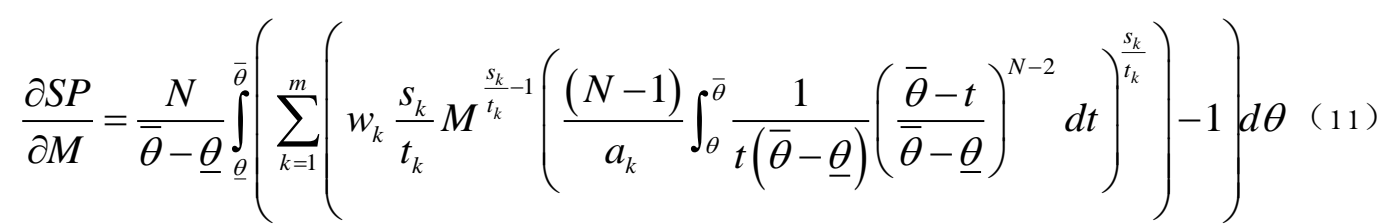

By observing Equation 11 you can find the value of the bonus that can't be directly obtained. But if $M=0,\left.\frac{\partial S P}{\partial M}\right|_{M=0}>0$; if $M \rightarrow \infty,\left.\frac{\partial S P}{\partial M}\right|_{M \rightarrow \infty}<0$. So there is at least one point in the interval $(0,+\infty)$ to satisfy $\frac{\partial S P}{\partial M}=0$. In order to determine if there is a unique optimal solution, take the second derivative of formula (10) with respect to $M$ as follow.

$$
\frac{\partial^{2} S P}{\partial M^{2}}=\frac{N}{\bar{\theta}-\underline{\theta}} \int_{\underline{\theta}}^{\bar{\theta}} \sum_{k=1}^{m}\left(\frac{w_{k} s_{k}}{t_{k}}\left(\frac{s_{k}}{t_{k}}-1\right) M^{\frac{s_{k}}{t_{k}}-2}\left(\frac{(N-1)}{a_{k}} \int_{\theta}^{\bar{\theta}} \frac{1}{t(\bar{\theta}-\underline{\theta})}\left(\frac{\bar{\theta}-t}{\bar{\theta}-\underline{\theta}}\right)^{N-2} d t\right)^{\frac{s_{k}}{t_{k}}}\right) d \theta
$$

Observing the above formula, we can find $\frac{\partial^{2} S P}{\partial M^{2}}<0$, this shows that the expected utility of the crowdsourcing has an optimal solution for the bonus. 


$$
\frac{N}{\bar{\theta}-\underline{\theta}} \int_{\underline{\theta}}^{\bar{\theta}}\left(\sum_{k=1}^{m}\left(w_{k} \frac{s_{k}}{t_{k}} M^{\frac{s_{k}}{t_{k}}-1}\left(\frac{(N-1)}{a_{k}} \int_{\theta}^{\bar{\theta}} \frac{1}{t(\bar{\theta}-\underline{\theta})}\left(\frac{\bar{\theta}-t}{\bar{\theta}-\underline{\theta}}\right)^{N-2} d t\right)^{\frac{s_{k}}{t_{k}}}\right)-1\right) d \theta=0
$$

The increase in the amount of bonuses in a certain range can motivate contestants to make more efforts for the crowdsourcing contest; but the bidder's own ability is limited, the bonus can't infinitely motivate the contestants, so the bonus should be set reasonably.

\section{Analysis of Crowdsourcing Contest Results}

Suppose an crowdsourcing publishes a crowdsourcing task and a bonus of $¥ 1,000$ on a crowdsourcing website. The crowdsourcing also announces the scoring rule and the lowest score. The crowdsourcing evaluates the bid from two aspects $\left(q_{1} 、 q_{2}\right)$. The weight given to the two attributes by the crowdsourcing is $W_{1}=5, W_{2}=3$, give the power of two attributes to $s_{1}=\frac{1}{2}, s_{2}=\frac{1}{3}$; The cost coefficient and power of the contestant for the two attributes $a_{1}=3, a_{2}=2, t_{1}=1, t_{2}=2$. The cost parameters of the contestant are uniformly distributed over the interval $[0.5,1.5]$. We separately influence the impact of the cost parameters and cost coefficient of the contestant on the bidding strategy. First consider the impact of the cost coefficient of the contestant on the bidding strategy of the contestant. As shown in Figure 1.

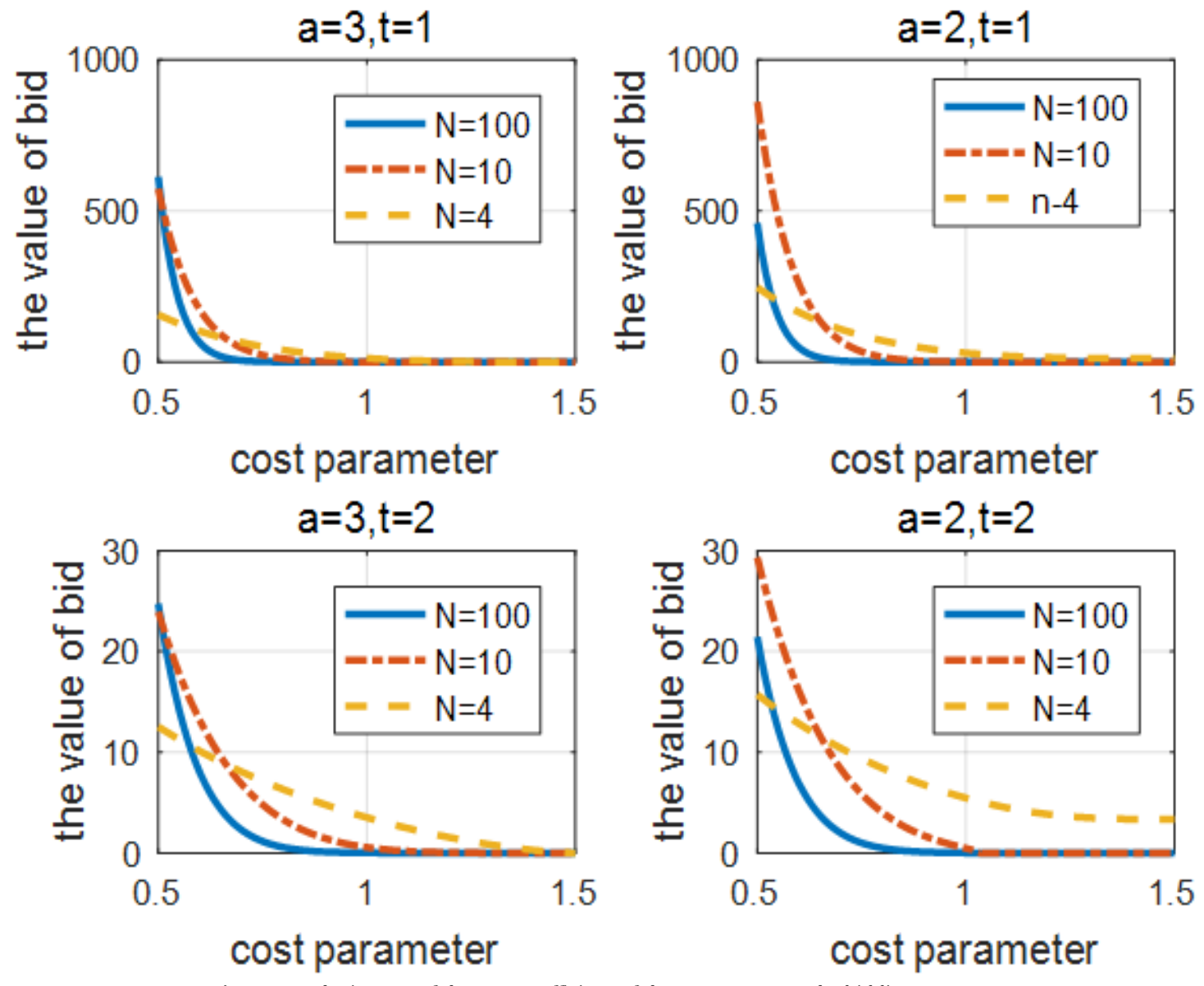

Figure-1. The impact of the cost coefficient of the contestant on the bidding strategy.

As can be seen from the figure, the greater the cost parameter of the contestant, the less effort the contestant will make on the bid. The cost coefficient of the contestant for the attribute and the power of the quality attribute have an effect on the bid of the attribute, the bidder's bid for this attribute is very sensitive to changes in $t_{k}$. The larger the cost coefficient of the attribute, the less effort the contestant will make on the attribute. 


\section{Conclusion}

Crowdsourcing has played an increasingly important role in enhancing corporate innovation capabilities and has become one of the research hotspots in academia. This paper studies the crowdsourcing contest under the fixed bonus mechanism. The purpose of the crowdsourcing is to maximize the sum of the quality of the bid submitted by the contestant. This paper builds a crowdsourcing model based on the multi-attribute reverse auction model to get the equilibrium bidding. Through research, it is found that the higher the cost parameter, the lower the expected utility of the contestant. In addition, the increase in the amount of the bonus can motivate the contestant to pay more for the bonus. However, because of the limitations of the ability of the contestant, the bonus amount is not unlimited for the incentive of the contestant. The discussion of bid、cost parameters and bonuses in this article is also for the crowdsourcing parties to better implement and utilize crowdsourcing.

\section{References}

Archak, N., \& Sundararajan, A. (2009). Optimal design of crowdsourcing contests. Paper presented at the ICIS 2009 Proceedings.

Chawla, S., Hartline, J. D., \& Sivan, B. (2015). Optimal crowdsourcing contests.

Che, Y. K., \& Gale, I. (2003). Optimal design of research contests. American Economic Review, 93(3), 646-671.

David, E., Azoulay-Schwartz, R., \& Kraus, S. (2002). Protocols and strategies for automated multi-attribute auctions. Paper presented at the Proceedings of the first International Joint Conference on Autonomous Agents and Multiagent Systems: Part 1. ACM.

Moldovanu, B., \& Sela, A. (2001). The optimal allocation of prizes in contests. American Economic Review, 91(3), $542-558$.

Morgan, J., \& Wang, R. (2010). Tournaments for ideas. California Management Review, 52(2), 77-97.

Schöttner, A. (2008). Fixed-prize tournaments versus first-price auctions in innovation contests. Economic Theory, 35(1), $57-71$.

Taylor, C. R. (1995). Digging for golden carrots: An analysis of research tournaments. American Economic Review, 85(2), 872-890.

Terwiesch, C., \& Xu, Y. (2008). Innovation contests, open innovation, and multiagent problem solving. Management Science, 54(9), 1529-1543.

Yang, Y., Chen, P. Y., \& Pavlou, P. A. (2009). Open innovation: An empirical study of online contests. Paper presented at the International Conference on Information Systems, Icis 2009, Phoenix, Arizona, Usa, December. DBLP. 